\title{
SunPy: A Python package for Solar Physics
}

Stuart J. Mumford ${ }^{* 1,2,3}$, Nabil Freij ${ }^{4}$, Steven Christe $^{5}$, Jack Ireland ${ }^{5}$, Florian Mayer ${ }^{6}$, V. Keith Hughitt ${ }^{7}$, Albert Y. Shih ${ }^{5}$, Daniel F. Ryan ${ }^{8,5}$, Simon Liedtke ${ }^{6}$, David Pérez-Suárez ${ }^{9}$, Pritish Chakraborty ${ }^{10}$, Vishnunarayan $\mathrm{K} \mathrm{I.}{ }^{6}$, Andrew Inglis ${ }^{11}$, Punyaslok Pattnaik ${ }^{12}$, Brigitta Sipőcz ${ }^{13}$, Rishabh Sharma ${ }^{6}$, Andrew Leonard ${ }^{3}$, David Stansby ${ }^{14}$, Russell Hewett ${ }^{15}$, Alex Hamilton ${ }^{6}$, Laura Hayes ${ }^{5}$, Asish Panda ${ }^{6}$, Matt Earnshaw $^{6}$, Nitin Choudhary ${ }^{16}$, Ankit Kumar ${ }^{6}$, Prateek Chanda ${ }^{17}$, Md Akramul Haque ${ }^{18}$, Michael S Kirk ${ }^{11}$, Michael Mueller ${ }^{6}$, Sudarshan Konge $^{6}$, Rajul Srivastava ${ }^{6}$, Yash Jain ${ }^{19}$, Samuel Bennett ${ }^{6}$, Ankit Baruah $^{6}$, Will Barnes ${ }^{20}$, Michael Charlton ${ }^{6}$, Shane Maloney ${ }^{21}$, Nicky Chorley $^{22}$, Himanshu ${ }^{6}$, Sanskar Modi ${ }^{6}$, James Paul Mason ${ }^{6}$, Naman9639 ${ }^{6}$, Jose Ivan Campos Rozo ${ }^{23}$, Larry Manley ${ }^{6}$, Agneet Chatterjee $^{24}$, John Evans ${ }^{6}$, Michael Malocha ${ }^{6}$, Monica G. Bobra ${ }^{25}$, Sourav Ghosh ${ }^{24}$, Airmansmith97 ${ }^{6}$, Dominik Stańczak ${ }^{26}$, Ruben De Visscher $^{6}$, Shresth Verma ${ }^{27}$, Ankit Agrawal ${ }^{6}$, Dumindu Buddhika ${ }^{6}$, Swapnil Sharma ${ }^{6}$, Jongyeob Park ${ }^{28}$, Matt Bates ${ }^{6}$, Dhruv Goel ${ }^{6}$, Garrison Taylor ${ }^{29}$, Goran Cetusic ${ }^{6}$, Jacob ${ }^{6}$, Mateo Inchaurrandieta ${ }^{6}$, Sally Dacie ${ }^{30}$, Sanjeev Dubey ${ }^{6}$, Deepankar Sharma ${ }^{6}$, Erik M. Bray ${ }^{6}$, Jai Ram Rideout ${ }^{31}$, Serge Zahniy ${ }^{5}$, Tomas Meszaros ${ }^{6}$, Abhigyan Bose ${ }^{6}$, André Chicrala ${ }^{32}$, Ankit ${ }^{6}$, Chloé Guennou ${ }^{6}$, Daniel D'Avella ${ }^{6}$, Daniel Williams $^{33}$, Jordan Ballew ${ }^{6}$, Nick Murphy ${ }^{34}$, Priyank Lodha ${ }^{6}$, Thomas Robitaille $^{6}$, Yash Krishan ${ }^{6}$, Andrew Hill ${ }^{6}$, Arthur Eigenbrot ${ }^{35}$, Benjamin Mampaey $^{36}$, Bernhard M. Wiedemann ${ }^{6}$, Carlos Molina ${ }^{6}$, Duygu Keșkek $^{6}$, Ishtyaq Habib ${ }^{6}$, Joseph Letts ${ }^{6}$, Juanjo Bazán ${ }^{37}$, Quinn Arbolante $^{38}$, Reid Gomillion ${ }^{6}$, Yash Kothari ${ }^{6}$, Yash Sharma ${ }^{6}$, Abigail L. Stevens $^{39}, 40$, Adrian Price-Whelan ${ }^{41}$, Ambar Mehrotra ${ }^{6}$, Arseniy Kustov $^{6}$, Brandon Stone ${ }^{6}$, Trung Kien Dang ${ }^{42}$, Emmanuel Arias ${ }^{6}$, Fionnlagh Mackenzie Dover ${ }^{1}$, Freek Verstringe ${ }^{36}$, Gulshan Kumar ${ }^{43}$, Harsh Mathur ${ }^{44}$, Igor Babuschkin ${ }^{6}$, Jaylen Wimbish ${ }^{6}$, Juan Camilo Buitrago-Casas $^{6}$, Kalpesh Krishna ${ }^{45}$, Kaustubh Hiware ${ }^{46}$, Manas Mangaonkar $^{6}$, Matthew Mendero ${ }^{6}$, Mickaël Schoentgen ${ }^{6}$, Norbert G Gyenge $^{47}$, Ole Streicher ${ }^{48}$, Rajasekhar Reddy Mekala ${ }^{6}$, Rishabh Mishra $^{6}$, Shashank Srikanth ${ }^{43}$, Sarthak Jain ${ }^{6}$, Tannmay Yadav ${ }^{49}$, Tessa D. Wilkinson ${ }^{6}$, Tiago M. D. Pereira ${ }^{50,51}$, Yudhik Agrawal ${ }^{12}$, jamescalixto $^{6}$, yasintoda ${ }^{6}$, and Sophie A. Murray ${ }^{52}$

1 SP2RC, School of Mathematics and Statistics, The University of Sheffield, UK 2 National Solar Observatory, 3665 Discovery Drive, Boulder, CO 803033 Aperio Software Ltd., Headingley Enterprise and Arts Centre, Bennett Road, Leeds LS6 3HN 4 Institute for Environmental Analytics, University of Reading, Reading RG6 6BX 5 NASA Goddard Space Flight Center, Greenbelt, MD 20771, USA 6 None 7 Center for Cancer Research, National Cancer Institute, Bethesda, MD 20892-9760, USA 8 American University, Washington, DC 20016, USA 9 University College London, Gower Street, London, UK 10 Manav Rachna University 11 Catholic University of America / NASA Goddard Space Flight Center, Greenbelt, MD 20771, USA 12 International Institute of Information 


\section{DOI: $10.21105 /$ joss. 01832}

\section{Software \\ - Review ¿ \\ - Repository ca \\ - Archive [ᄌ}

Editor: Arfon Smith ש Reviewers:

- Omwcraig

- @ceb8

Submitted: 07 October 2019 Published: 14 February 2020

\section{License}

Authors of papers retain copyright and release the work under a Creative Commons Attribution 4.0 International License (CC-BY).

In partnership with

\section{A A AMERICAN ASTRONOMICAL SOCIETY}

This article and software are linked with research article DOI 10.3847/1538-4357/ab4f7a, published in the The Astrophysical Journal.
Technology, Hyderabad 500 032, India 13 DIRAC Institute, Department of Astronomy, University of Washington, Seattle, WA 98195, USA 14 Mullard Space Science Laboratory, University College London, Surrey, UK 15 Department of Mathematics, Virginia Polytechnic Institute and State University, Blacksburg, VA 24061-0123, USA 16 Department of Mathematics, Indian Institute of Technology, Kharagpur, 721302, India 17 Department of Computer Science \& Technology, Indian Institute of Engineering Science \& Technology, Shibpur, 711103, India 18 Department of Mechanical Engineering, ZHCET, Aligarh Muslim University, Aligarh, India 19 Indian Institute of Technology, Kharagpur, 721302, India 20 Lockheed Martin Solar and Astrophysics Laboratory / Bay Area Environmental Research Institute 21 Trinity College Dublin / Dublin Institute for Advanced Studies 22 Centre for Fusion, Space and Astrophysics, Physics Department, University of Warwick, Coventry CV4 7AL, United Kingdom 23 Institut für Physik/IGAM - Karl-Franzens University of Graz, Austria 24 Jadavpur University, Kolkata 25 W.W. Hansen Experimental Physics Laboratory, Stanford University, Stanford, CA 94305, USA 26 University of Warsaw 27 ABV-Indian Institute of Information Technology and Management, Gwalior, MP 474015, India 28 Space Science Division, Korea Astronomy and Space Science Institute, Daejeon 34055, South Korea 29

Harvard-Smithsonian Center for Astrophysics, Cambridge, MA 02138, USA 30 Mullard Space Science Laboratory, University College London, Holmbury St. Mary, Surrey, RH5 6NT, UK 31 Dogfox Software LLC, Flagstaff, AZ 86001, USA 32 Northumbria University, England 33 SUPA, University of Glasgow, Glasgow G12 8QQ, United Kingdom 34 Center for Astrophysics | Harvard \& Smithsonian 35 National Solar Observatory 36 Royal Observatory of Belgium 37 CIEMAT, Astroparticle physics, Madrid, Spain. 38 Lockheed Martin Solar and Astrophysics Laboratory 39 Department of Physics \& Astronomy, Michigan State University, 567 Wilson Road, East Lansing, MI 48824, USA 40 Department of Astronomy, University of Michigan, 1085 South University Avenue, Ann Arbor, MI 48109, USA 41 Center for Computational Astrophysics, Flatiron Institute, 162 Fifth Ave, New York, NY 10010, USA 42 Saw Swee Hock School of Public Health, National University Health System, National University of Singapore, Singapore 43 International Institute of Information Technology, Hyderabad $\mathbf{4 4}$ Indian Institute of Astrophysics, Bangalore $\mathbf{4 5}$ University of Massachusetts, Amherst 46 Indian Institute of Technology, Kharagpur 47 SP2RC, School of Mathematics and Statistics, University of Sheffield, Sheffield, S3 7RH, UK 48 Leibniz Institute for Astrophysics Potsdam, Germany 49 Department of Chemical Engineering, Indian Institute of Technology Kharagpur, 721302, India 50 Rosseland Centre for Solar Physics, University of Oslo, P.O. Box 1029 Blindern, NO-0315 Oslo, Norway 51 Institute of Theoretical Astrophysics, University of Oslo, P.O. Box 1029 Blindern, NO-0315 Oslo, Norway 52 Trinity College Dublin, Ireland

\section{Summary}

The Sun, our nearest star, is a local laboratory for studying universal physical processes. Solar physics as a discipline includes studying the Sun both as a star and as the primary driver of space weather throughout the heliosphere. Due to the Sun's proximity, the temporal and spatial resolution of solar observations are orders of magnitude larger than those of other stars. This leads to significant differences in the data-analysis software needs of solar physicists compared with astrophysicists.

The sunpy Python package is a community-developed, free, and open-source solar data analysis environment for Python. It is managed by the SunPy Project, an organization that facilitates and promotes the use of open development and open source packages like sunpy through community engagement and tools such as GitHub, mailing lists, and matrix.

The four most significant subpackages of sunpy are described below.

The sunpy.net subpackage provides a unified interface that simplifies and homogenizes search and retrieval by querying and downloading data from many solar data sources, irrespective of the underlying data-source client. It currently supports sourcing data from 18 different spaceand ground-based solar observatories.

The sunpy .map and sunpy. timeseries subpackages provide core data types (Map and Time Series, respectively) that are designed to provide a general, standard, and consistent interface

\footnotetext{
*The author list in this paper is sorted by number of commits to the core SunPy repository.
} 
for loading and representing solar data across different instruments and missions. These classes load data which conform to solar physics standards and conventions such as FITS (Wells, Greisen, \& Harten, 1981), FITS World Coordinate Systems (WCS) (Greisen \& Calabretta, 2002), and solar-specific FITS headers (Thompson, 2006), while allowing customization to account for differences in specific instruments. Visualization methods are also provided to inspect and plot those data. Example visulizations of both TimeSeries and Map are shown in Figure 1.
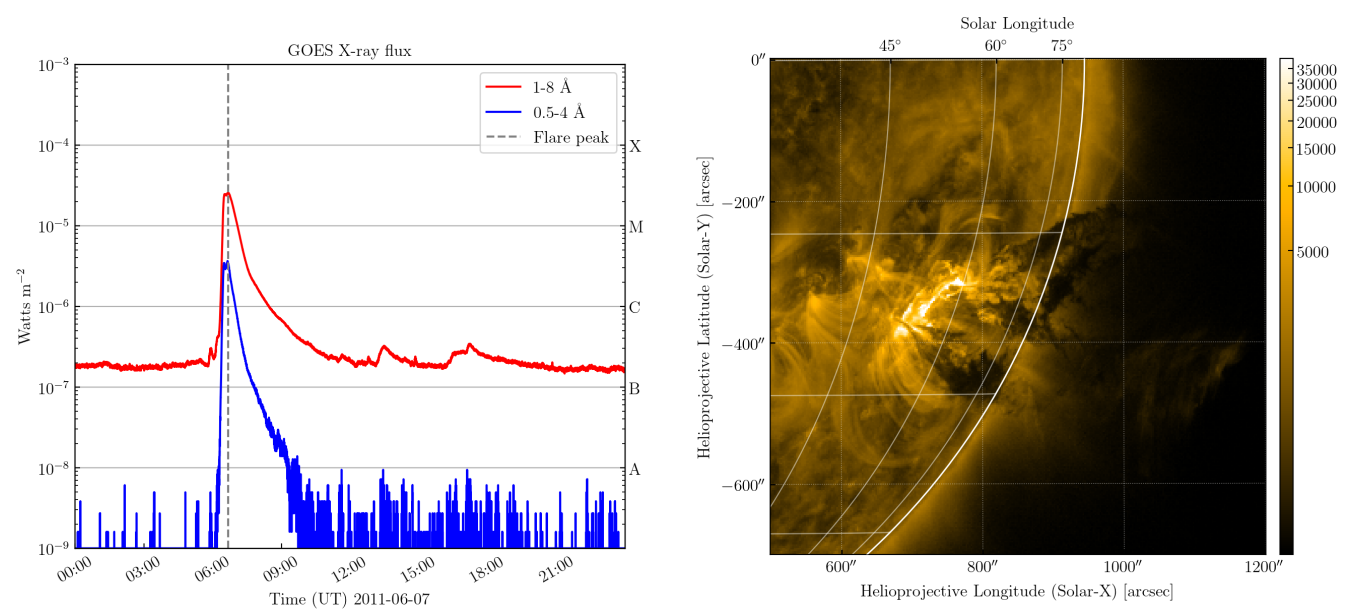

Figure 1: Left: An example of TimeSeries for the GOES X-ray Sensor in two broadband channels. Right: A Map of the extreme ultraviolet $171 \AA$ channel of AIA corresponding to the time of a solar flare depicted by the vertical dashed line in the left-hand panel.

The sunpy.coordinates subpackage provides support for representing and transforming coordinates used in solar physics and astrophysics. These coordinates may represent events (e.g., flares), features on or above the Sun (e.g., magnetic loops), or the position of structures traveling throughout the heliosphere (e.g., coronal mass ejections). The package currently implements the most widely used Sun-centered coordinate frames, and extends astropy.co ordinates.

Other functionality provided by sunpy includes physical models of solar behavior, such as differential rotation, color maps for certain data sources, image-processing routines integrated with Map, and useful physical parameters such as constants.

The sunpy package is designed to be extensible, which means that it is easy to add support for additional instruments or data sources. It relies heavily on the astropy (The Astropy Collaboration et al., 2018) Python package as well as the scientific python stack (e.g. numpy (van der Walt, Colbert, \& Varoquaux, 2011), scipy (Jones, Oliphant, Peterson, \& others, n.d.), matplotlib (Hunter, 2007) and pandas (McKinney, 2010)).

A more complete description of the SunPy Project and the sunpy package, including the methodology, development model, and implementation, as well as a comparison with other commonly-used packages in solar physics, can be found in (Barnes et al., 2020).

The SunPy Project supports affiliated packages, which build upon or extend the functionality of sunpy. The current affiliated packages are drms (Glogowski, Bobra, Choudhary, Amezcua, \& Mumford, 2019), ndcube, radiospectra and IRISPy. The Project is also a member of the Python in Heliophysics community (PyHC, Annex et al., 2018), whose mission is to enable interdisciplinary analysis across all sub-disciplines of heliophysics by adhering to standards for code development and interoperability. 


\section{Acknowledgements}

SunPy is a NumFOCUS sponsored package.

\section{References}

Annex, A., Alterman, B. L., Azari, A., Barnes, W., Bobra, M., Cecconi, B., Christe, S., et al. (2018). Python in heliophysics community (pyhc) standards. doi:10.5281/zenodo. 2529131

Barnes, W. T., Bobra, M. G., Christe, S. D., Freij, N., Hayes, L. A., Ireland, J., Mumford, S., et al. (2020). The sunpy project: Open source development and status of the version 1.0 core package. The Astrophysical Journal, 890(1), 68. doi:10.3847/1538-4357/ab4f7a

Glogowski, K., Bobra, M., Choudhary, N., Amezcua, A., \& Mumford, S. (2019). Drms: A python package for accessing hmi and aia data. Journal of Open Source Software, 4(40), 1614. doi: $10.21105 /$ joss. 01614

Greisen, E. W., \& Calabretta, M. R. (2002). Representations of world coordinates in fits, 395, 1061-1075. doi:10.1051/0004-6361:20021326

Hunter, J. D. (2007). Matplotlib: A 2D graphics environment. Computing in Science \& Engineering, 9(3), 90-95. doi:10.1109/MCSE.2007.55

Jones, E., Oliphant, T., Peterson, P., \& others. (n.d.). SciPy: Open source scientific tools for Python. Retrieved from http://www.scipy.org/

McKinney, W. (2010). Data structures for statistical computing in python. In S. van der Walt \& J. Millman (Eds.), Proceedings of the 9th python in science conference (pp. 51-56).

The Astropy Collaboration, Price-Whelan, A. M., Sipőcz, B. M., Günther, H. M., Lim, P. L., Crawford, S. M., Conseil, S., et al. (2018). The astropy project: Building an openscience project and status of the v2.0 core package. The Astronomical Journal, 156(3), 123. doi:10.3847/1538-3881/aabc4f

Thompson, W. T. (2006). Coordinate systems for solar image data. Astronomy and Astrophysics, 449, 791-803. doi:10.1051/0004-6361:20054262

van der Walt, S., Colbert, S. C., \& Varoquaux, G. (2011). The numpy array: A structure for efficient numerical computation. Computing in Science Engineering, 13(2), 22-30. doi:10.1109/MCSE.2011.37

Wells, D. C., Greisen, E. W., \& Harten, R. H. (1981). FITS - a Flexible Image Transport System. Astronomy and Astrophysics Supplement Series, 44, 363. 